\title{
Fuzzy Monte Carlo Finite Element Solution Applied in Engineering Structures
}

\author{
Bo LIU ${ }^{\mathrm{a}}$, Changhong LIU ${ }^{\mathrm{b}}$, Hehui WANG, Rong JING, Ciwei XU, Hongjun QI \\ ( Key Laboratory of Pressure Systems and Safety, Ministry of Education, East China University \\ of Science and Technology, Shanghai, 200237, China ) \\ a13122006197@163.com, bliuch1977@qq.com
}

Key words: Fuzzy; Monte carlo; Finite element method; Assembly flanged joint.

\begin{abstract}
Based on the theory of the fuzziness and stochastic finite element solution, a fuzzy monte carlo finite element solution was presented. Firstly, with the $\lambda$-cut set of the fuzzy set, fuzzy parameters in a fuzzy engineering structure were transformed into stochastic variables in the uniform distribution function. Secondly, the structure was solved by the monte carlo finite element method. Thirdly, according to the fuzzy expansion and decomposition theorem, the results of a fuzzy engineering structure were obtained. Finally, the sensitivity analysis was applied to reduce solution time. Numerical results of the fuzzy assembly flanged joint structure shown that the solution was efficient.
\end{abstract}

\section{Introduction}

Many engineering structures are lack defined and imprecisely known to be modeled and analyzed by conventional deterministic procedures [1]. Analysis of such systems requires the use of fuzzy information, that is, information that is vague, imprecise, qualitative, linguistic or incomplete. In a structural analysis, the fuzzy or imprecise information may be present in the geometry, material properties, applied load and boundary conditions [2]. The need to incorporate subjective information into the design and analysis of engineering systems has led to the development of fuzzy finite element analysis.

In this work, a practical approach for analyzing the response of structures with fuzzy parameters is developed. The methodology that is presented in this paper involves stochastic finite element method and the fuzzy analysis procedures. The uncertainties in the temperature and structural parameters are represented using fuzzy sets. Once the combinations are obtained, the monte carlo finite element method is then carried out to determine the extreme values of the responses. These quantities are used to construct the membership of the response.

In the following sections, a brief summary of the fuzzy monte carlo finite element methodology is provided. This is followed by an overview of the fuzzy computational strategy.

\section{Fuzzy monte carlo finite element method}

According to the fuzzy set [3], when the fuzzy parameters $\tilde{p}$ in the structural system, the fuzzy finite element equation of the structure can be expressed as $\mathrm{K}(\tilde{\mathrm{p}}) \mathrm{u}=\mathrm{F}(\tilde{\mathrm{p}}), \mathrm{K}$ as the global stiffness matrix, $F$ as the external load vector, $u$ as the displacement vector. When $\tilde{p}=\tilde{p}(\lambda)$ as the parameter vector, according to the fuzzy extension and decomposition theorem, the formula can be written as $K\left(\tilde{p}_{\lambda}\right) u=F\left(\tilde{p}_{\lambda}\right) . \quad \tilde{p}_{\lambda}$ is the interval variables $\left[p_{\lambda_{L}}, p_{\lambda_{U}}\right]$ under determining level of $\lambda$ cut set. 
The membership functions of fuzzy variable $P$ on $\lambda$ cut set interval $\left[p_{\lambda_{L}}, p_{\lambda_{U}}\right]$ can be expressed as:

$$
f_{p_{\lambda}}=\left\{\begin{array}{cc}
1, & X \in\left[P_{\lambda_{L}}, P_{\lambda_{U}}\right] \\
0, & \text { others }
\end{array}\right.
$$

Then there is an equivalent uniform distribution function in the corresponding stochastic space, the probability density function:

$$
p(y)=\frac{1}{p_{\lambda_{U}}-p_{\lambda_{L}}}
$$

According to the theory of probability and statistics, this variable can be viewed as a stochastic variable uniformly distributed on the interval $\left[p_{\lambda_{L}}, p_{\lambda_{U}}\right]$.

Therefore, the fuzzy finite element equation can be transformed into a stochastic finite element equation, which is subject to the uniform distribution parameters in the interval of the $\lambda$-cut set. The process is as follows:

(1) To determine the $\lambda$ numerical values in $\lambda \in[0,1]$;

(2) The $\lambda$-cut level is applied to the fuzzy variables;

(3) According to the expansion theorem, the interval fuzzy finite element equations under $\lambda$ cut sets can be transformed into stochastic finite element equations which is subject to a uniform distribution in the $\lambda$-cut level intervals;

(4) The $\lambda$-cut level interval fuzzy finite element equations are solved by the stochastic finite element method;

(5) With the fuzzy decomposition theorem, the results' membership functions of the fuzzy finite element model are determined by results of the stochastic finite element method in the different $\lambda$ cut sets;

For the random structures, the monte carlo finite element method is a popular and effective method. Monte Carlo PDS method in ANSYS can not only achieve the reliability analysis of the structure, also its sensitivity analysis module can determine the input variables which have a significant influence on the structural response of output. Through the sensitivity analysis to determine the input variables of great influence on the output variable, reduced number of the input variables of less effective, verification of error in two fuzzy output solutions by recalculation.

\section{Assembly flanged joint}

Flange has a very important role in pressure vessels and piping systems, while the assembly flanged joint is applied in many fields as one of the most widely used sealing device. A calculation of an assembly flanged joint will be cited as the calculation case, calculation process and specific parameters detailed in the literature [4].As shown in Fig.1, an assembly flanged joint is composed of 24-inch flange, 8-inch flange, pipe, manholes and bolts, gaskets and other components. 


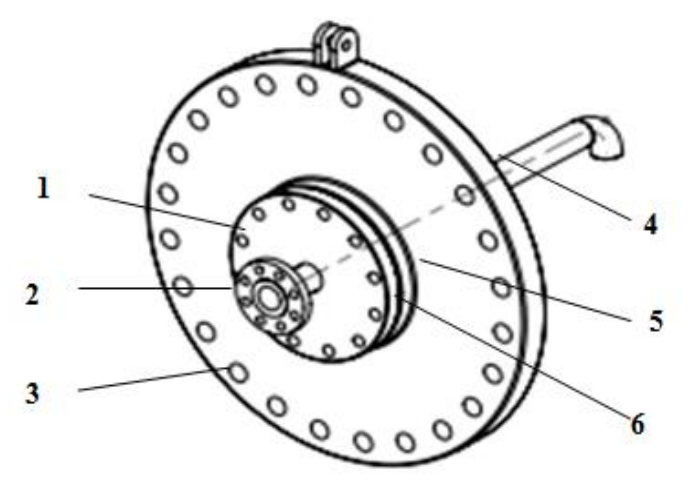

1. 8-inch flange 2. Pipes 3. Bolt holes 4. Manhole takeover

5. 24-inch flange 6. Gasket

Fig.1 Schematic diagram of an assembly flanged joint

Calculation of the flange and sealing evaluation is mainly conducted for preload conditions and operating conditions. Two groups of bolt preload F1,F2 is applied to the flange when in preload condition, the internal medium pressure of the assembly flanged pipes is $1.4 \mathrm{MPa}$, and the internal medium pressure of the flange is set to $\mathrm{P}$, environment temperature is set to $20{ }^{\circ} \mathrm{C}$, the operating temperature is set to T. According to the above method, fuzzy input variables should be designed, in this case, the fuzzy input variables are: the operating temperature $\mathrm{T}$, the internal medium pressure $\mathrm{P}$, and two sets of bolt preload F1, F2, and Young's modulus E1 of nuts, Young's modulus E2 of bolts, Young's modulus E3 of flanges. Then, the graphs of triangle membership function of all fuzzy input variables are obtained through MATLAB software program, where applied different $\lambda$ cut sets, and get the range of all fuzzy input variables at different $\lambda$ cut set.

Table 1 Interval distribution of partial fuzzy variables in different $\lambda$ cut sets

\begin{tabular}{|c|c|c|c|c|c|c|c|c|}
\hline & \multicolumn{2}{|c|}{$\mathrm{T}\left({ }^{\circ} \mathrm{C}\right)$} & \multicolumn{2}{|l|}{$\mathrm{F} 1(\mathrm{~N})$} & \multicolumn{2}{|l|}{$\mathrm{F} 2(\mathrm{~N})$} & \multicolumn{2}{|c|}{$\mathrm{P}(\mathrm{MPa})$} \\
\hline$\lambda=0$ & {$[420$} & 580] & {$[15000$} & 17000] & {$[55000$} & 65000] & {$[0.5$} & $1.5]$ \\
\hline$\lambda=0.1$ & {$[428$} & 572] & {$[15100$} & 16900] & {$[55500$} & 64500] & {$[0.55$} & $1.45]$ \\
\hline$\lambda=0.3$ & {$[444$} & $556]$ & {$[15300$} & 16700] & {$[56500$} & 63500] & {$[0.65$} & $1.35]$ \\
\hline$\lambda=0.5$ & {$[460$} & 540] & {$[15500$} & 16500] & {$[57500$} & 62500] & {$[0.75$} & $1.25]$ \\
\hline$\lambda=0.7$ & {$[476$} & 556] & {$[15700$} & 16300] & {$[58500$} & 61500] & {$[0.85$} & $1.15]$ \\
\hline$\lambda=0.9$ & {$[492$} & 508] & [15900 & 16100] & [59500 & 60500] & {$[0.95$} & $1.05]$ \\
\hline$\lambda=1$ & 500 & & 16000 & & 60000 & & 1 & \\
\hline
\end{tabular}

\section{Computation solution}

According to the sealing indicators of the flange, taken deflection angle of 8-inch flange and 24-inch flange, gasket minimum stress of 8-inch flange and 24-inch flange as the fuzzy output variables. In ANSYS probability design module (PDS), the seven fuzzy input variables are defined as uniformly distributed stochastic input variables, using the Monte Carlo simulation of sampling methods, sampling frequency of 48 , get the fuzzy results of all output variables of different $\lambda$ cut sets, the part of fuzzy solution are shown in table 2 . 
Table2 The fuzzy results of output variables

\begin{tabular}{|c|c|c|c|c|c|c|c|c|}
\hline \multirow{2}{*}{$\begin{array}{l}\text { Cut } \\
\text { sets }\end{array}$} & \multicolumn{2}{|c|}{$\begin{array}{c}\text { 24-inchflange } \\
\text { deflection angle }\end{array}$} & \multicolumn{2}{|c|}{$\begin{array}{c}\text { 8-inch flange } \\
\text { deflection angle }\end{array}$} & \multicolumn{2}{|c|}{$\begin{array}{c}\text { 24-inch flange } \\
\text { gasket minimum } \\
\text { stress } \\
\end{array}$} & \multicolumn{2}{|c|}{$\begin{array}{c}\text { 8-inch flange } \\
\text { gasket minimum } \\
\text { stress }\end{array}$} \\
\hline & {$[0.75 \mathrm{E}-3$} & $1.42 \mathrm{E}-3]$ & {$[0.31 \mathrm{E}-3$} & $0.91 \mathrm{E}-3]$ & {$[-52.03$} & $-29.31]$ & {$[-24.20$} & $-20.24]$ \\
\hline$\lambda=0.1$ & {$[0.76 \mathrm{E}-3$} & $1.42 \mathrm{E}-3]$ & {$[0.35 \mathrm{E}-3$} & $0.84 \mathrm{E}-3]$ & {$[-49.87$} & $-31.42]$ & {$[-23.82$} & $-20.53]$ \\
\hline$\lambda=0.3$ & {$[0.83 \mathrm{E}-3$} & $1.33 \mathrm{E}-3]$ & {$[0.40 \mathrm{E}-3$} & 0.77 E-3] & {$[-47.74$} & $-33.54]$ & {$[-23.39$} & -20.89] \\
\hline$\lambda=0.5$ & [0.89E-3 & $1.23 \mathrm{E}-3]$ & {$[0.44$ E-3 } & 0.70 E-3] & {$[-45.63$} & $-35.46]$ & {$[-23.02$} & -21.19] \\
\hline$\lambda=0.7$ & {$[0.94 \mathrm{E}-3$} & $1.13 \mathrm{E}-3]$ & {$[0.48 \mathrm{E}-3$} & 0.66 E-3] & {$[-43.85$} & $-36.94]$ & {$[-22.65$} & $-21.73]$ \\
\hline$\lambda=0.9$ & {$[1.02 \mathrm{E}-3$} & $1.09 \mathrm{E}-3]$ & {$[0.53 \mathrm{E}-3$} & $0.58 \mathrm{E}-3]$ & {$[-41.29$} & $-38.97]$ & {$[-22.31$} & $-21.96]$ \\
\hline$\lambda=1$ & $1.05 \mathrm{E}-3$ & & $0.56 \mathrm{E}-3$ & & -40.25 & & -22.13 & \\
\hline
\end{tabular}

By calculation, the results of seven fuzzy output variables will also obey the triangle distribution. The membership functions of fuzzy output variables are triangle membership function. The maximum possible values of the 8-inch flange deflection angle and 24-inch flange deflection angle are $0.556 \mathrm{E}-3^{\circ}$ and $1.051 \mathrm{E}-3^{\circ}$,the minimum possible values of 8 -inch flange gasket and 24 -inch flange gasket are $-22.134 \mathrm{MP}$ and $-40.247 \mathrm{MPa}$.
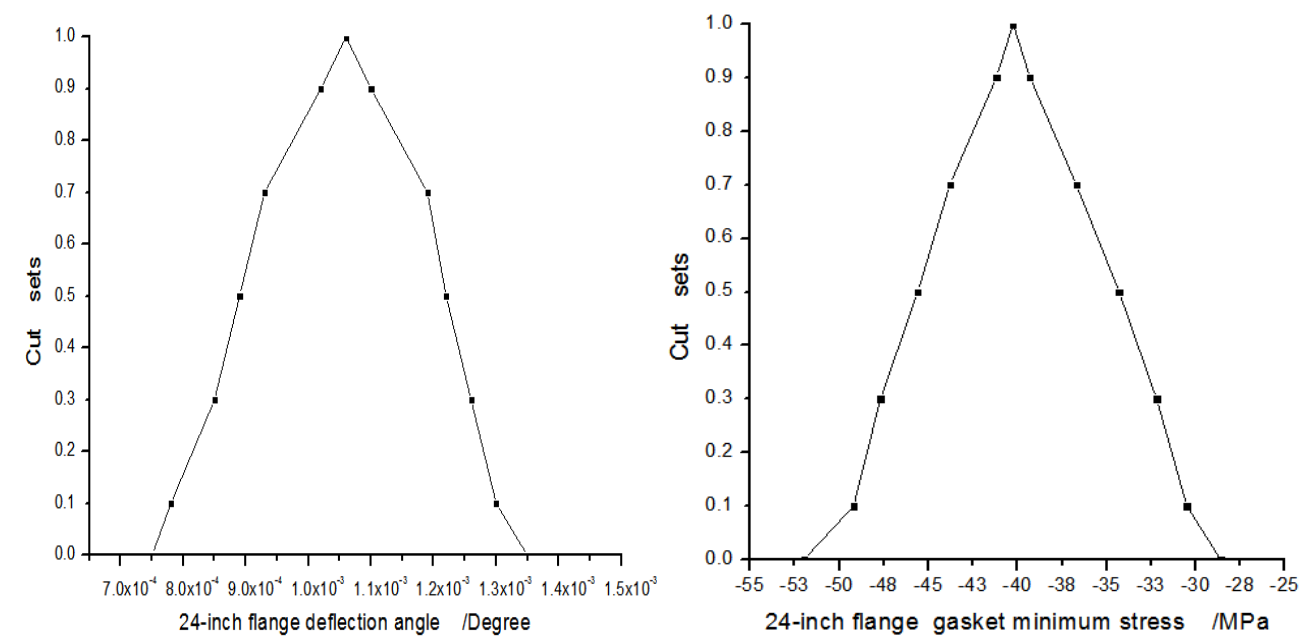

Fig.2 The membership functions of the assembly flanged joint

\section{Sensitivity analysis}

When the cut set $\lambda=0.5$, the sensitivity analysis result of 24 -inch flange gasket minimum stress is shown in Fig. 3, the main affecting factors contain Young's modules of flange and operating pressure. Remove of the fuzzy input variables of no significant effect on 24-inch flange gasket minimum stress, include temperature T, two bolt preload F1, F2, nuts and bolts Young's modulus E1, E2, calculate again with only two effective fuzzy input variables: operating pressure $\mathrm{P}$ and Young's modules of flange E3. 


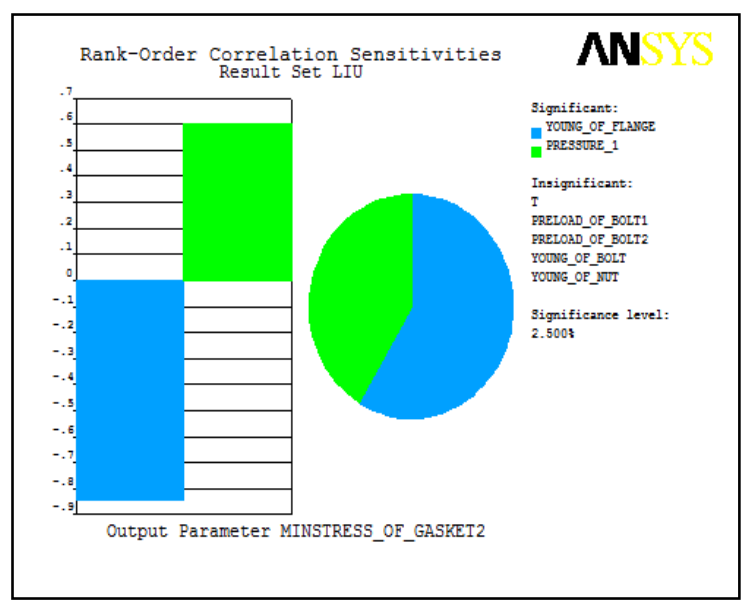

Fig.3 The sensitivity result of 24-inch flange gasket minimum stress $(\lambda=0.5)$

Comparison of the results of the two operations, it was approximately same when removed the fuzzy input variables with no significant effect on the fuzzy output variables, and the latter error was less $3 \%$ than the former, but the work of recalculation significantly reduced. The comparison is listed in Table 3.

Table3 Two finite element computation results of part fuzzy output variables $\quad(\lambda=0.5)$

\begin{tabular}{cccc}
\hline Calculation times & Cut set & $\begin{array}{c}\text { 24-inch flange gasket minimum stress } \\
(\mathrm{MPa})\end{array}$ \\
\hline NO.1 & $\lambda=0.5$ & {$[-45.63$} & $-35.46]$ \\
NO.2 & $\lambda=0.5$ & {$[-44.51$} & $-35.15]$ \\
\hline
\end{tabular}

\section{Conclusion}

This paper presents a fuzzy monte carlo finite element solution that can convert a fuzzy variable to an equivalent stochastic variable. According to the fuzzy expansion and decomposition theorem, convert the interval fuzzy finite element equations under the corresponding $\lambda$ cut set to the equivalent finite element equations of uniform distribution of equivalent interval, solve fuzzy structure by using stochastic finite element method and combined with the sensitivity analysis to obtain the fuzzy input variables has a significant effect on the output [5]. The paper example has shown that the method is simple and effective, and can improve efficiency and ensure solution accuracy of the optimal design of engineering structure design.

\section{References}

[1] D. Moens, D. Vandepitte, Fuzzy finite element method for frequency response function analysis of uncertain structures, International Journal for Numerical Methods in Engineering, 63 (2004) 2480-2507.

[2] F.S. Arnold, Fuzzy Random Variables, J. Insurance: Mathematics and Economics 44 (2009) 307-314. 
[3] R.L. Muhanna, H. Zhang, R. Mullen, Interval Finite Elements as a Basis for Generalized Models of Uncertainty in Engineering Mechanics Mathematics, Reliable computing, 13 (2007) 173-194.

[4] H.H. Wang, J.C. Lu, K.S. Guan, Z.W. Wang, Strength and seal FE analysis of combined flanges with a pipe, Pressure vessel Technology, 29 (2012) 22-29.

[5] C.H. Liu, Q. Chen, Interval analysis of fuzzy-random heat conduction in composite, Applied mathematics and mechanics, 26 (2005) 1312-1318. 